\title{
Article \\ On the Reciprocal Sums of Products of Two Generalized Bi-Periodic Fibonacci Numbers
}

\author{
Younseok Choo $\mathbb{D}$
}

Citation: Choo, Y. On the Reciprocal Sums of Products of Two Generalized Bi-Periodic Fibonacci Numbers. Mathematics 2021, 9, 178. https:// doi.org/10.3390/math9020178

Received: 20 December 2020 Accepted: 15 January 2021 Published: 17 January 2021

Publisher's Note: MDPI stays neutral with regard to jurisdictional clai$\mathrm{ms}$ in published maps and institutional affiliations.

Copyright: (C) 2021 by the author. Licensee MDPI, Basel, Switzerland. This article is an open access article distributed under the terms and conditions of the Creative Commons Attribution (CC BY) license (https:// creativecommons.org/licenses/by/ $4.0 /)$.
Department of Electronic and Electrical Convergence Engineering, Hongik University, Sejong-Ro 2639, Sejong 30016, Korea; yschoo@hongik.ac.kr

Abstract: This paper concerns the properties of the generalized bi-periodic Fibonacci numbers $\left\{G_{n}\right\}$ generated from the recurrence relation: $G_{n}=a G_{n-1}+G_{n-2}$ ( $n$ is even) or $G_{n}=b G_{n-1}+G_{n-2}$ ( $n$ is odd). We derive general identities for the reciprocal sums of products of two generalized biperiodic Fibonacci numbers. More precisely, we obtain formulas for the integer parts of the numbers $\left(\sum_{k=n}^{\infty} \frac{(a / b)^{\tilde{\xi}(k+1)}}{G_{k} G_{k+m}}\right)^{-1}, m=0,2,4, \cdots$, and $\left(\sum_{k=n}^{\infty} \frac{1}{G_{k} G_{k+m}}\right)^{-1}, m=1,3,5, \cdots$

Keywords: bi-periodic Fibonacci numbers; reciprocal; floor function

MSC: 11B37; 11B39

\section{Introduction}

As is well known, the Fibonacci sequence $\left\{F_{n}\right\}$ is generated from the recurrence relation $F_{n}=F_{n-1}+F_{n-2}(n \geq 2)$ with the initial conditions $F_{0}=0$ and $F_{1}=1$. The Fibonacci numbers possess many interesting properties and appear in a variety of application fields [1].

Many authors tried to generalize the Fibonacci sequence. For example, Falcon and Plaza [2] considered the $k$-Fibonacci sequence. Edson and Yayenie [3] introduced the bi-periodic Fibonacci sequence $\left\{f_{n}\right\}$ defined by

$$
f_{0}=0, f_{1}=1, \quad f_{n}=\left\{\begin{array}{ll}
a f_{n-1}+f_{n-2}, & \text { if } n \in \mathbb{N}_{e} ; \\
b f_{n-1}+f_{n-2}, & \text { if } n \in \mathbb{N}_{o},
\end{array} \quad(n \geq 2),\right.
$$

where $\mathbb{N}_{e}\left(\mathbb{N}_{o}\right.$, respectively) denotes the set of positive even (odd, respectively) integers. Filipponi [4] defined the incomplete Fibonacci sequence, and Ramírez [5] introduced the bi-periodic incomplete Fibonacci sequence.

In the remainder of this paper, we use the notation $\left\{G_{n}\right\}_{n=0}^{\infty}=S\left(G_{0}, G_{1}, a, b\right)$ to denote the generalized bi-periodic Fibonacci numbers $\left\{G_{n}\right\}$ generated from the recurrence relation

$$
G_{n}=\left\{\begin{array}{ll}
a G_{n-1}+G_{n-2}, & \text { if } n \in \mathbb{N}_{e} ; \\
b G_{n-1}+G_{n-2}, & \text { if } n \in \mathbb{N}_{o},
\end{array} \quad(n \geq 2),\right.
$$

with initial conditions $G_{0}$ and $G_{1}$, where $G_{0}$ is a nonnegative integer, $G_{1}, a$ and $b$ are positive integers.

Recently, Ohtsuka and Nakamura [6] reported an interesting property of the Fibonacci numbers $\left\{F_{n}\right\}=S(0,1,1,1)$ and proved the following identities:

$$
\begin{aligned}
& \left\lfloor\left(\sum_{k=n}^{\infty} \frac{1}{F_{k}}\right)^{-1}\right\rfloor= \begin{cases}F_{n}-F_{n-1}, & \text { if } n \geq 2 \text { and } n \in \mathbb{N}_{e} \\
F_{n}-F_{n-1}-1, & \text { if } n \geq 1 \text { and } n \in \mathbb{N}_{o},\end{cases} \\
& \left\lfloor\left(\sum_{k=n}^{\infty} \frac{1}{F_{k}^{2}}\right)^{-1}\right\rfloor= \begin{cases}F_{n-1} F_{n}-1, & \text { if } n \geq 2 \text { and } n \in \mathbb{N}_{e} \\
F_{n-1} F_{n}, & \text { if } n \geq 1 \text { and } n \in \mathbb{N}_{o},\end{cases}
\end{aligned}
$$


where $\lfloor\cdot\rfloor$ is the floor function.

Following the work of Ohtsuka and Nakamura, diverse results for the numbers of the form $\left\{G_{n}\right\}=S\left(G_{0}, G_{1}, a, a\right)$ have been reported in the literature (see [7-15] and references cited therein).

On the other hand, reciprocal sums of the generalized bi-periodic numbers were considered in [16,17]. In [16], Basbuk and Yazlik proved the following identity for $\left\{G_{n}\right\}=S(0,1, a, b)$ :

$$
\left\lfloor\left(\sum_{k=n}^{\infty} \frac{(a / b)^{\psi(k)}}{G_{k}}\right)^{-1}\right\rfloor= \begin{cases}G_{n}-G_{n-1}, & \text { if } n \geq 2 \text { and } n \in \mathbb{N}_{e} \\ G_{n}-G_{n-1}-1, & \text { if } n \geq 1 \text { and } n \in \mathbb{N}_{o}\end{cases}
$$

where

$$
\psi(k)=\xi(k+1)-\xi(n+1)-(-1)^{n}\left\lfloor\frac{k-n}{2}\right\rfloor,
$$

and $\xi(n)$ is the parity function, such that

$$
\xi(n)=\left\{\begin{array}{l}
0, \text { if } n \in\{0\} \cup \mathbb{N}_{e} \\
1, \text { if } n \in \mathbb{N}_{0}
\end{array}\right.
$$

For $\left\{G_{n}\right\}=S\left(G_{0}, G_{1}, a, b\right)$, Choi and Choo [17] identified the integer parts for the numbers

$$
\left(\sum_{k=n}^{\infty} \frac{(a / b)^{\xi(k+1)}}{G_{k}^{2}}\right)^{-1}
$$

In this paper, we extend the results in [17] by considering the reciprocal sums of products of two generalized bi-periodic Fibonacci numbers. More precisely, we obtain general identities for the numbers

$$
\left(\sum_{k=n}^{\infty} \frac{(a / b)^{\tau}(k+1)}{G_{k} G_{k+m}}\right)^{-1}, m \in\{0\} \cup \mathbb{N}_{e},
$$

and

$$
\left(\sum_{k=n}^{\infty} \frac{1}{G_{k} G_{k+m}}\right)^{-1}, m \in \mathbb{N}_{0}
$$

\section{Results}

2.1. The Case where $m \in\{0\} \cup \mathbb{N}_{e}$

Lemma 1 below will be used to prove the results for the case where $m \in \mathbb{N}_{e}$.

Lemma 1. Assume that $m \in\{0\} \cup \mathbb{N}_{e}$. Then, for $\left\{G_{n}\right\}=S\left(G_{0}, G_{1}, a, b\right)$, (a)-(e) below hold:

(a) $G_{n+2} G_{n+m+1}-G_{n} G_{n+m-1}=a^{\xi(n)} b^{\xi(n+1)} G_{n} G_{n+m}+a^{\tilde{\xi}(n+1)} b^{\xi^{(n)}} G_{n+1} G_{n+m+1}$.

(b) $G_{n+1} G_{n+m}-G_{n+2} G_{n+m-1}=(-1)^{n}\left(G_{m} G_{3}-G_{m+1} G_{2}\right)$.

(c) $a^{\xi(n+1)} b^{\xi(n)} G_{n-1} G_{n+m+1}-a^{\xi(n)} b^{\tilde{\xi}(n+1)} G_{n} G_{n+m}=(-1)^{n}\left(a G_{m+1} G_{1}-b G_{m+2} G_{0}\right)$.

(d) $G_{n+1} G_{n+m-2}-G_{n} G_{n+m-1}=(-1)^{n}\left(G_{m} G_{3}-G_{m+1} G_{2}\right)$.

(e) $G_{n-1} G_{n+m}-G_{n} G_{n+m-1}=(-1)^{n}\left(G_{m} G_{1}-G_{m+1} G_{0}\right)$.

Proof. Since

$$
G_{n}=a^{\tilde{\xi}(n-1)} b^{\tilde{\xi}(n)} G_{n-1}+G_{n-2}
$$

then, (a) follows from the identity

$$
\begin{aligned}
G_{n} G_{n+m+1} & =\left(G_{n+2}-a^{\tilde{\zeta}(n+1)} b^{\tilde{\zeta}(n)} G_{n+1}\right) G_{n+m+1} \\
& =G_{n}\left(a^{\tilde{\zeta}(n)} b^{\xi(n+1)} G_{n+m}+G_{n+m-1}\right) .
\end{aligned}
$$


(b)-(e) are special cases of ([18], Theorem 2.2).

Theorem 1. Consider the generalized bi-periodic Fibonacci numbers $\left\{G_{n}\right\}=S\left(G_{0}, G_{1}, a, b\right)$ and let

$$
\Phi_{m}:=b\left(G_{m} G_{3}-G_{m+1} G_{2}\right) .
$$

If $m \in\{0\} \cup \mathbb{N}_{e}$, then (a) and (b) below hold:

(a) If

$$
\frac{\Phi_{m}}{a b+2} \notin \mathbb{Z},
$$

define

$$
g_{m}:=\left\lfloor\frac{\Phi_{m}}{a b+2}\right\rfloor+\Delta
$$

where

$$
\Delta=\left\{\begin{array}{l}
1, \text { if } \Phi_{m}>0 \\
0, \text { if } \Phi_{m}<0
\end{array}\right.
$$

(i) If $\Phi_{m}>0$, then there exist positive integers $n_{0}$ and $n_{1}$ such that

$$
\left\lfloor\left(\sum_{k=n}^{\infty} \frac{\left(\frac{a}{b}\right)^{\xi(k+1)}}{G_{k} G_{k+m}}\right)^{-1}\right\rfloor= \begin{cases}b G_{n} G_{n+m-1}+g_{m}-1, & \text { if } n \geq n_{0} \text { and } n \in \mathbb{N}_{e} \\ b G_{n} G_{n+m-1}-g_{m}, & \text { if } n \geq n_{1} \text { and } n \in \mathbb{N}_{o} .\end{cases}
$$

(ii) If $\Phi_{m}<0$, then there exist positive integers $n_{2}$ and $n_{3}$ such that

$$
\left\lfloor\left(\sum_{k=n}^{\infty} \frac{\left(\frac{a}{b}\right)^{\xi(k+1)}}{G_{k} G_{k+m}}\right)^{-1}\right\rfloor= \begin{cases}b G_{n} G_{n+m-1}+g_{m}, & \text { if } n \geq n_{2} \text { and } n \in \mathbb{N}_{e} \\ b G_{n} G_{n+m-1}-g_{m}-1, & \text { if } n \geq n_{3} \text { and } n \in \mathbb{N}_{o} .\end{cases}
$$

(b) If

$$
\frac{\Phi_{m}}{a b+2} \in \mathbb{Z}
$$

define

$$
\hat{g}_{m}:=\frac{\Phi_{m}}{a b+2}
$$

and

$$
\Gamma_{m}:=\hat{g}_{m} b\left(b G_{m+2} G_{0}-a G_{m+1} G_{1}\right)-\hat{g}_{m}^{2} .
$$

(i) If $\Gamma_{m} \geq 0$, then there exist positive integers $n_{4}$ and $n_{5}$, such that

$$
\left\lfloor\left(\sum_{k=n}^{\infty} \frac{\left(\frac{a}{b}\right)^{\xi(k+1)}}{G_{k} G_{k+m}}\right)^{-1}\right\rfloor= \begin{cases}b G_{n} G_{n+m-1}+\hat{g}_{m}, & \text { if } n \geq n_{4} \text { and } n \in \mathbb{N}_{e} ; \\ b G_{n} G_{n+m-1}-\hat{g}_{m}, & \text { if } n \geq n_{5} \text { and } n \in \mathbb{N}_{0} .\end{cases}
$$

(ii) If $\Gamma_{m}<0$, then there exist positive integers $n_{4}$ and $n_{5}$, such that

$$
\left\lfloor\left(\sum_{k=n}^{\infty} \frac{\left(\frac{a}{b}\right)^{\xi(k+1)}}{G_{k} G_{k+m}}\right)^{-1}\right\rfloor= \begin{cases}b G_{n} G_{n+m-1}+\hat{g}_{m}-1, & \text { if } n \geq n_{6} \text { and } n \in \mathbb{N}_{e} \\ b G_{n} G_{n+m-1}-\hat{g}_{m}-1, & \text { if } n \geq n_{7} \text { and } n \in \mathbb{N}_{0} .\end{cases}
$$

Proof. (a) To prove (5), assume that $\Phi_{m}>0$. Then

$$
\Phi_{m}-g_{m}(a b+2)<0 .
$$


Firstly, consider

$$
\begin{aligned}
X_{1} & =\frac{1}{b G_{n} G_{n+m-1}+(-1)^{n} g_{m}}-\frac{1}{b G_{n+2} G_{n+m+1}+(-1)^{n} g_{m}}-\frac{\left(\frac{a}{b}\right)^{\xi(n+1)}}{G_{n} G_{n+m}}-\frac{\left(\frac{a}{b}\right)^{\xi(n)}}{G_{n+1} G_{n+m+1}} \\
& =\frac{Y_{1}}{\left(b G_{n} G_{n+m-1}+(-1)^{n} g_{m}\right)\left(b G_{n+2} G_{n+m+1}+(-1)^{n} g_{m}\right) G_{n} G_{n+1} G_{n+m} G_{n+m+1}}
\end{aligned}
$$

where, by Lemma 1 (a)

$$
Y_{1}=\left\{\left(\frac{a}{b}\right)^{\xi(n)} G_{n} G_{n+m}+\left(\frac{a}{b}\right)^{\xi(n+1)} G_{n+1} G_{n+m+1}\right\} \hat{Y}_{1}
$$

with

$$
\hat{Y}_{1}=b^{2}\left(G_{n} G_{n+1} G_{n+m} G_{n+m+1}-G_{n} G_{n+2} G_{n+m-1} G_{n+m+1}\right)-(-1)^{n} g_{m} b\left(G_{n} G_{n+m-1}+G_{n+2} G_{n+m+1}\right)-g_{m}^{2} .
$$

By Lemma $1(b, c)$, we have

$$
\begin{aligned}
G_{n} G_{n+1} G_{n+m} G_{n+m+1}-G_{n} G_{n+2} G_{n+m-1} G_{n+m+1} & =\left(G_{n+1} G_{n+m}-G_{n+2} G_{n+m-1}\right) G_{n} G_{n+m+1} \\
& =(-1)^{n}\left(G_{m} G_{3}-G_{m+1} G_{2}\right) G_{n} G_{n+m+1}
\end{aligned}
$$

and

$$
\begin{aligned}
& G_{n} G_{n+m-1}+G_{n+2} G_{n+m+1} \\
= & G_{n}\left(G_{n+m+1}-a^{\xi(n+m)} b^{\tilde{\xi}(n+m+1)} G_{n+m}\right)+\left(a^{\xi(n+1)} b^{\xi(n)} G_{n+1}+G_{n}\right) G_{n+m+1} \\
= & 2 G_{n} G_{n+m+1}-a^{\xi(n)} b^{\tau(n+1)} G_{n} G_{n+m}+a^{\xi(n+1)} b^{\xi(n)}\left(a^{\xi(n)} b^{\xi(n+1)} G_{n}+G_{n-1}\right) G_{n+m+1} \\
= & (a b+2) G_{n} G_{n+m+1}-a^{\xi(n)} b^{\tau(n+1)} G_{n} G_{n+m}+a^{\xi(n+1)} b^{\xi(n)} G_{n-1} G_{n+m+1} \\
= & (a b+2) G_{n} G_{n+m+1}+(-1)^{n}\left(a G_{m+1}-b G_{m+2} G_{0}\right) .
\end{aligned}
$$

Then

$$
\begin{aligned}
\hat{Y}_{1}= & (-1)^{n} b^{2}\left(G_{m} G_{3}-G_{m+1} G_{2}\right) G_{n} G_{n+m+1} \\
& -(-1)^{n} g_{m} b\left\{(a b+2) G_{n} G_{n+m+1}+(-1)^{n}\left(a G_{m+1}-b G_{m+2} G_{0}\right)\right\}-g_{m}^{2} \\
= & (-1)^{n} b\left\{\Phi_{m}-g(a b+2)\right\} G_{n} G_{n+m+1}+g_{m} b\left(b G_{m+2} G_{0}-a G_{m+1} G_{1}\right)-g_{m}^{2} .
\end{aligned}
$$

If $n \in \mathbb{N}_{e}$, then there exists a positive integer $m_{0}$ such that, for $n \geq m_{0}, X_{1}<0$, and

$$
\frac{1}{b G_{n} G_{n+m-1}+g_{m}}-\frac{1}{b G_{n+2} G_{n+m+1}+g_{m}}<\frac{\left(\frac{a}{b}\right)^{\xi(n+1)}}{G_{n} G_{n+m}}+\frac{\left(\frac{a}{b}\right)^{\xi(n)}}{G_{n+1} G_{n+m+1}} .
$$

Repeatedly applying the above inequality, we have

$$
\frac{1}{b G_{n} G_{n+m-1}+g_{m}}<\sum_{k=n}^{\infty} \frac{\left(\frac{a}{b}\right)^{\xi(k+1)}}{G_{k} G_{k+m}}, \text { if } n \geq m_{0} \text { and } n \in \mathbb{N}_{e} .
$$

Similarly, we obtain, for some positive integer $m_{1}$,

$$
\sum_{k=n}^{\infty} \frac{\left(\frac{a}{b}\right)^{\xi(k+1)}}{G_{k} G_{k+m}}<\frac{1}{b G_{n} G_{n+m-1}-g_{m}}, \text { if } n \geq m_{1} \text { and } n \in \mathbb{N}_{o} .
$$

Next, consider 


$$
\begin{aligned}
X_{2}= & \frac{1}{b G_{n} G_{n+m-1}+(-1)^{n} g_{m}-1}-\frac{1}{b G_{n+1} G_{n+m}+(-1)^{n+1} g_{m}-1}-\frac{\left(\frac{a}{b}\right)^{\xi(n+1)}}{G_{n} G_{n+m}} \\
= & \frac{Y_{2}}{\left(b G_{n} G_{n+m-1}+(-1)^{n} g_{m}-1\right)\left(b G_{n+1} G_{n+m}+(-1)^{n+1} g_{m}-1\right) G_{n} G_{n+m}}, \\
\quad \text { where } & \\
Y_{2}= & b G_{n} G_{n+1} G_{n+m}^{2}-a^{\tilde{\zeta}(n+1)} b^{1+\xi(n)} G_{n} G_{n+1} G_{n+m-1} G_{n+m}-b G_{n}^{2} G_{n+m-1} G_{n+m+1} \\
& -(-1)^{n} g_{m}\left\{2 G_{n} G_{n+m}+a^{\xi(n+1)} b^{\xi(n)}\left(G_{n+1} G_{n+m}-G_{n} G_{n+m-1}\right)\right\} \\
& +a^{\xi(n+1)} b^{\xi(n)}\left(G_{n} G_{n+m-1}+G_{n+1} G_{n+m}\right)+a^{\xi(n+1)} b^{\xi(n)-1}\left(g_{m}^{2}-1\right) .
\end{aligned}
$$

Using Lemma $1(\mathrm{~d}, \mathrm{e})$, we have

$$
\begin{aligned}
& b G_{n} G_{n+1} G_{n+m}^{2}-a^{\xi(n+1)} b^{1+\xi^{(n)}} G_{n} G_{n+1} G_{n+m-1} G_{n+m}-b G_{n}^{2} G_{n+m-1} G_{n+m} \\
= & b G_{n} G_{n+1} G_{n+m}\left(G_{n+m}-a^{\xi(n+1)} b^{\xi(n)} G_{n+m-1}\right)-b G_{n}^{2} G_{n+m-1} G_{n+m} \\
= & b G_{n} G_{n+m}\left(G_{n+1} G_{n+m-2}-G_{n} G_{n+m-1}\right) \\
= & (-1)^{n} b\left(G_{m} G_{3}-G_{m+1} G_{2}\right) G_{n} G_{n+m} \\
& \quad \text { and } \\
& 2 G_{n} G_{n+m}+a^{\xi(n+1)} b^{\xi(n)}\left(G_{n+1} G_{n+m}-G_{n} G_{n+m-1}\right) \\
= & \left.2 G_{n} G_{n+m}+a^{\xi(n+1)} b^{\xi(n)}\left(a^{\xi(n)} b^{\xi(n+1)} G_{n}+G_{n-1}\right) G_{n+m}-a^{\xi(n+1)} b^{\xi(n)} G_{n} G_{n+m-1}\right) \\
= & (a b+2) G_{n} G_{n+m}+a^{\xi(n+1)} b^{\xi(n)}\left(G_{n-1} G_{n+m}-G_{n} G_{n+m-1}\right) \\
= & (a b+2) G_{n} G_{n+m}+(-1)^{n} a^{\xi(n+1)} b^{\tilde{\xi}(n)}\left(G_{m} G_{1}-G_{m+1} G_{0}\right) .
\end{aligned}
$$

Hence we obtain

$$
\begin{aligned}
Y_{2}= & (-1)^{n}\left\{\Phi_{m}-g_{m}(a b+2)\right\} G_{n} G_{n+m}+a^{\xi(n+1)} b^{\xi(n)}\left(G_{n} G_{n+m-1}+G_{n+1} G_{n+m}\right) \\
& -a^{\xi(n+1)} b^{\xi(n)} g_{m}\left(G_{m} G_{1}-G_{m+1} G_{0}\right)+a^{\xi(n+1)} b^{\xi(n)-1}\left(g_{m}^{2}-1\right),
\end{aligned}
$$

and there exists a positive integer $m_{2}$ such that, for $n \geq m_{2}, X_{2}>0$, and

$$
\frac{\left(\frac{a}{b}\right)^{\xi(n+1)}}{G_{n} G_{n+m}}<\frac{1}{b G_{n} G_{n+m-1}+(-1)^{n} g_{m}-1}-\frac{1}{b G_{n+1} G_{n+m}+(-1)^{n+1} g_{m}-1} .
$$

Repeatedly applying the above inequality, we have

$$
\sum_{k=n}^{\infty} \frac{\left(\frac{a}{b}\right)^{\xi(k+1)}}{G_{k} G_{k+m}}<\frac{1}{b G_{n} G_{n+m-1}+(-1)^{n} g_{m}-1}, \text { if } n \geq m_{2} .
$$

Similarly, consider

$$
\begin{aligned}
X_{3} & =\frac{1}{b G_{n} G_{n+m-1}+(-1)^{n} g_{m}+1}-\frac{1}{b G_{n+1} G_{n+m}+(-1)^{n+1} g_{m}+1}-\frac{\left(\frac{a}{b}\right)^{\xi(n+1)}}{G_{n} G_{n+m}} \\
& =\frac{Y_{3}}{\left(b G_{n} G_{n+m-1}+(-1)^{n} g_{m}+1\right)\left(b G_{n+1} G_{n+m}+(-1)^{n+1} g_{m}+1\right) G_{n} G_{n+m}},
\end{aligned}
$$

where 


$$
\begin{aligned}
Y_{3}= & Y_{2}-2 a^{\xi(n+1)} b^{\xi(n)}\left(G_{n} G_{n+m-1}+G_{n+1} G_{n+m}\right) \\
= & (-1)^{n}\left\{\Phi_{m}-g_{m}(a b+2)\right\} G_{n} G_{n+m}-a^{\xi(n+1)} b^{\xi(n)}\left(G_{n} G_{n+m-1}+G_{n+1} G_{n+m}\right) \\
& -a^{\xi(n+1)} b^{\tilde{\xi}(n)} g_{m}\left(G_{m} G_{1}-G_{m+1} G_{0}\right)+a^{\xi(n+1)} b^{\tilde{\zeta}(n)-1}\left(g_{m}^{2}-1\right) .
\end{aligned}
$$

There exists a positive integer $m_{3}$ such that, for $n \geq m_{3}, X_{3}<0$, and

$$
\frac{1}{b G_{n} G_{n+m-1}+(-1)^{n} g_{m}+1}-\frac{1}{b G_{n+1} G_{n+m}+(-1)^{n+1} g_{m}+1}<\frac{\left(\frac{a}{b}\right)^{\xi(n+1)}}{G_{n} G_{n+m}},
$$

from which we have

$$
\frac{1}{b G_{n} G_{n+m-1}+(-1)^{n} g_{m}+1}<\sum_{k=n}^{\infty} \frac{\left(\frac{a}{b}\right)^{\xi(k+1)}}{G_{k} G_{k+m}}, \text { if } n \geq m_{3} .
$$

Then (5) follows from (9)-(12).

Now, suppose that $\Phi_{m}<0$. In this case, we have

$$
\Phi_{m}-g_{m}(a b+2)>0,
$$

and (9)-(12) are respectively modified as

$$
\begin{aligned}
& \sum_{k=n}^{\infty} \frac{\left(\frac{a}{b}\right)^{\xi(k+1)}}{G_{k} G_{k+m}}<\frac{1}{b G_{n} G_{n+m-1}+g_{m}}, \text { if } n \geq m_{4} \text { and } n \in \mathbb{N}_{e}, \\
& \frac{1}{b G_{n} G_{n+m-1}-g_{m}}<\sum_{k=n}^{\infty} \frac{\left(\frac{a}{b}\right)^{\xi(k+1)}}{G_{k} G_{k+m}}, \text { if } n \geq m_{5} \text { and } n \in \mathbb{N}_{o}, \\
& \sum_{k=n}^{\infty} \frac{\left(\frac{a}{b}\right)^{\xi(k+1)}}{G_{k} G_{k+m}}<\frac{1}{b G_{n} G_{n+m-1}+(-1)^{n} g_{m}-1}, \text { if } n \geq m_{6},
\end{aligned}
$$

and

$$
\frac{1}{b G_{n} G_{n+m-1}+(-1)^{n} g_{m}+1}<\sum_{k=n}^{\infty} \frac{\left(\frac{a}{b}\right)^{\xi^{(k+1)}}}{G_{k} G_{k+m}} \text { if } n \geq m_{7} .
$$

Then, (6) easily follows and the proof of (a) is completed.

(b) Suppose that

$$
\frac{\Phi_{m}}{a b+2} \in \mathbb{Z} .
$$

We recall the proof of (a). If $\Gamma_{m} \geq 0$, then replacing $g_{m}$ by $\hat{g}_{m}$, we have $\hat{Y}_{1}=\Gamma_{m} \geq 0$, and there exists a positive integer $m_{8}$ such that $X_{1}>0$ if $n \geq m_{8}$. Hence, we obtain

$$
\sum_{k=n}^{\infty} \frac{\left(\frac{a}{b}\right)^{\xi(k+1)}}{G_{k} G_{k+m}}<\frac{1}{b G_{n} G_{n+m-1}+(-1)^{n} \hat{g}_{m}}, \text { if } n \geq m_{8} .
$$

Similarly, there exists a positive integer $m_{9}$ such that $X_{3}<0$ if $n \geq m_{9}$, from which we have

$$
\frac{1}{b G_{n} G_{n+m}+(-1)^{n} \hat{g}+1}<\sum_{k=n}^{\infty} \frac{\left(\frac{a}{b}\right)^{\xi(k+1)}}{G_{k} G_{k+m}} \text {, if } n \geq m_{9} .
$$


Then, (7) follows from (17) and (18). (8) can be proved similarly, and details are omitted.

If $a=b$, then Theorem 1 reduces ([7], Theorem 2.1) with $m \in\{0\} \cup \mathbb{N}_{e}$ and $\left\{G_{n}\right\}=$ $\left\{H_{n}\right\}=S\left(G_{0}, G_{1}, a, a\right)$.

If $m=0$, then Theorem 1 reduces ([17], Theorem 2).

\subsection{The Case Where $m \in \mathbb{N}_{o}$}

To deal with the case where $m \in \mathbb{N}_{0}$, we need the following lemma.

Lemma 2. Assume that $m \in \mathbb{N}_{0}$. Then, for $\left\{G_{n}\right\}=S\left(G_{0}, G_{1}, a, b\right)$, (a)-(e) below hold:
(a) $G_{n+2} G_{n+m+1}-G_{n} G_{n+m-1}=a^{\xi(n+1)} b^{\xi(n)}\left(G_{n} G_{n+m}+G_{n+1} G_{n+m+1}\right)$.
(b) $a^{\tilde{\xi}(n+1)} b^{\tilde{\xi}(n)} G_{n+1} G_{n+m}-a^{\xi(n)} b^{\tilde{\xi}(n+1)} G_{n+2} G_{n+m-1}=(-1)^{n}\left(a G_{m} G_{3}-b G_{m+1} G_{2}\right)$.
(c) $G_{n-1} G_{n+m+1}-G_{n} G_{n+m}=(-1)^{n}\left(G_{m+2} G_{0}-G_{m+1} G_{1}\right)$.
(d) $a^{\xi(n+1)} b^{\xi(n)} G_{n+1} G_{n+m-2}-a^{\xi(n)} b^{\xi(n+1)} G_{n} G_{n+m-1}=(-1)^{n}\left(a G_{m} G_{3}-b G_{m+1} G_{2}\right)$.
(e) $a^{\xi(n+1)} b^{\tilde{\xi}(n)} G_{n-1} G_{n+m}-a^{\xi(n)} b^{\xi(n+1)} G_{n} G_{n+m-1}=(-1)^{n}\left(a G_{m} G_{1}-b G_{m+1} G_{0}\right)$.

Proof. (a) follows from the identity

$$
\begin{aligned}
G_{n} G_{n+m+1} & =\left(G_{n+2}-a^{\xi(n+1)} b^{\tilde{\xi}(n)} G_{n+1}\right) G_{n+m+1} \\
& =G_{n}\left(a^{\xi(n+m)} b^{\xi(n+m+1)} G_{n+m}+G_{n+m-1}\right) .
\end{aligned}
$$

(b)-(e) are special cases of ([18], Theorem 2.2).

Theorem 2. Consider the generalized bi-periodic Fibonacci numbers $\left\{G_{n}\right\}=S\left(G_{0}, G_{1}, a, b\right)$ and let

$$
\Delta_{m}:=a G_{m} G_{3}-b G_{m+1} G_{2} .
$$

If $m \in \mathbb{N}_{0}$, then (a) and (b) below hold:

(a) If

$$
\frac{\Delta_{m}}{a b+2} \notin \mathbb{Z}
$$

define

$$
h_{m}:=\left\lfloor\frac{\Delta_{m}}{a b+2}\right\rfloor+\Delta,
$$

where

$$
\Delta=\left\{\begin{array}{l}
1, \text { if } \Delta_{m}>0 \\
0, \text { if } \Delta_{m}<0
\end{array}\right.
$$

(i) If $\Delta_{m}>0$, then there exist positive integers $n_{0}$ and $n_{1}$ such that

$$
\left\lfloor\left(\sum_{k=n}^{\infty} \frac{1}{G_{k} G_{k+m}}\right)^{-1}\right\rfloor= \begin{cases}b G_{n} G_{n+m-1}+h_{m}-1, & \text { if } n \geq n_{0} \text { and } n \in \mathbb{N}_{e} \\ a G_{n} G_{n+m-1}-h_{m}, & \text { if } n \geq n_{1} \text { and } n \in \mathbb{N}_{o}\end{cases}
$$

(ii) If $\Delta_{m}<0$, then there exist positive integers $n_{2}$ and $n_{3}$ such that

$$
\left\lfloor\left(\sum_{k=n}^{\infty} \frac{1}{G_{k} G_{k+m}}\right)^{-1}\right\rfloor= \begin{cases}b G_{n} G_{n+m-1}+h_{m}, & \text { if } n \geq n_{2} \text { and } n \in \mathbb{N}_{e} \\ a G_{n} G_{n+m-1}-h_{m}-1, & \text { if } n \geq n_{3} \text { and } n \in \mathbb{N}_{o}\end{cases}
$$

(b) If

$$
\frac{\Delta_{m}}{a b+2} \in \mathbb{Z},
$$


define

$$
\hat{h}_{m}:=\frac{\Delta_{m}}{a b+2},
$$

and

$$
\Psi_{m}:=\hat{h}_{m} a b\left(G_{m+1} G_{1}-G_{m+2} G_{0}\right)-\hat{h}_{m}^{2} .
$$

(i) If $\Psi_{m} \geq 0$, then there exist positive integers $n_{4}$ and $n_{5}$ such that

$$
\left\lfloor\left(\sum_{k=n}^{\infty} \frac{1}{G_{k} G_{k+m}}\right)^{-1}\right\rfloor=\left\{\begin{array}{l}
a G_{n} G_{n+m-1}+\hat{h}_{m}, \text { if } n \geq n_{4} \text { and } n \in \mathbb{N}_{e} ; \\
b G_{n} G_{n+m-1}-\hat{h}_{m}, \text { if } n \geq n_{5} \text { and } n \in \mathbb{N}_{o} .
\end{array}\right.
$$

(ii) If $\Psi_{m}<0$, then there exist positive integers $n_{6}$ and $n_{7}$ such that

$$
\left\lfloor\left(\sum_{k=n}^{\infty} \frac{1}{G_{k} G_{k+m}}\right)^{-1}\right\rfloor=\left\{\begin{array}{l}
a G_{n} G_{n+m-1}+\hat{h}_{m}-1, \text { if } n \geq n_{6} \text { and } n \in \mathbb{N}_{e} ; \\
b G_{n} G_{n+m-1}-\hat{h}_{m}-1, \text { if } n \geq n_{7} \text { and } n \in \mathbb{N}_{o} .
\end{array}\right.
$$

Proof. (a) To prove (19), assume that $\Delta_{m}>0$. Then

$$
\Delta_{m}-h_{m}(a b+2)<0 .
$$

Firstly, consider

$$
\begin{aligned}
& X_{1}=\frac{1}{a^{\tau}(n) b^{\tau}(n+1) G_{n} G_{n+m-1}+(-1)^{n} h_{m}}-\frac{1}{a^{\tau}(n) b^{\tau}(n+1) G_{n+2} G_{n+m+1}+(-1)^{n} h_{m}}-\frac{1}{G_{n} G_{n+m}}-\frac{1}{G_{n+1} G_{n+m+1}}
\end{aligned}
$$

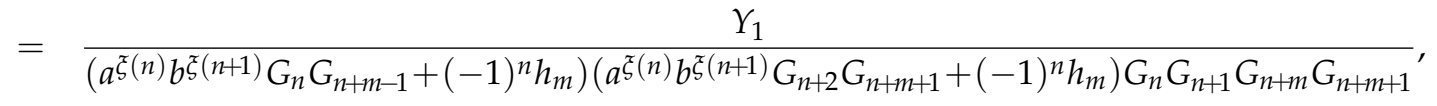

where, by Lemma 2 (a)

$$
Y_{1}=\left(G_{n} G_{n+m}+G_{n+1} G_{n+m+1}\right) \hat{Y}_{1}
$$

with

$$
\begin{aligned}
\hat{Y}_{1}= & a b G_{n} G_{n+1} G_{n+m} G_{n+m+1}-a^{2 \tilde{\xi}(n)} b^{2 \tilde{\xi}(n+1)} G_{n} G_{n+2} G_{n+m-1} G_{n+m+1} \\
& -(-1)^{n} h_{m} a^{\tilde{\zeta}(n)} b^{\tilde{\xi}(n+1)}\left(G_{n} G_{n+m-1}+G_{n+2} G_{n+m+1}\right)-h_{m}^{2} .
\end{aligned}
$$

By Lemma $2(\mathrm{~b}, \mathrm{c})$, we have

$$
\begin{aligned}
& a b G_{n} G_{n+1} G_{n} G_{n+m}-a^{2 \xi(n)} b^{2 \xi(n+1)} G_{n} G_{n+2} G_{n+m-1} G_{n+m+1} \\
& =\left(a b G_{n+1} G_{n+m}-a^{2 \xi(n)} b^{2 \xi(n+1)} G_{n+2} G_{n+m-1}\right) G_{n} G_{n+m+1} \\
& =a^{\xi(n)} b^{\xi^{(n+1)}}\left(a^{\xi(n+1)} b^{\xi(n)} G_{n+1} G_{n+m}-a^{\xi(n)} b^{\xi(n+1)} G_{n+2} G_{n+m-1}\right) G_{n} G_{n+m+1} \\
& =(-1)^{n} a^{\xi^{(n)}} b^{\xi(n+1)}\left(a G_{m} G_{3}-b G_{m+1} G_{2}\right) G_{n} G_{n+m+1} \text {, } \\
& G_{n} G_{n+m-1}+G_{n+2} G_{n+m+1} \\
& =G_{n}\left(G_{n+m+1}-a^{\xi(n+m)} b^{\tilde{\xi}(n+m+1)} G_{n+m}\right)+\left(a^{\tilde{\xi}(n+1)} b^{\tilde{\xi}(n)} G_{n+1}+G_{n}\right) G_{n+m+1} \\
& =2 G_{n} G_{n+m+1}-a^{\xi(n+1)} b^{\xi(n)} G_{n} G_{n+m}+a^{\xi(n+1)} b^{\xi(n)}\left(a^{\xi(n)} b^{\xi(n+1)} G_{n}+G_{n-1}\right) G_{n+m+1} \\
& =(a b+2) G_{n} G_{n+m+1}+a^{\xi^{(n+1)}} b^{\xi^{(n)}}\left(G_{n-1} G_{n+m+1}-G_{n} G_{n+m}\right) \\
& =(a b+2) G_{n} G_{n+m+1}+(-1)^{n} a^{\xi(n+1)} b^{\xi(n)}\left(G_{m+2} G_{0}-G_{m+1} G_{1}\right) \text {. }
\end{aligned}
$$

Then 


$$
\begin{aligned}
\hat{Y}_{1}= & (-1)^{n} a^{\xi^{(n)}} b^{\xi(n+1)}\left(a G_{m} G_{3}-b G_{m+1} G_{2}\right) G_{n} G_{n+m+1} \\
& -(-1)^{n} h_{m} a^{\xi^{(n)}} b^{\xi(n+1)}\left\{(a b+2) G_{n} G_{n+m+1}+(-1)^{n} a^{\xi^{(n+1)}} b^{\xi^{(n)}}\left(G_{m+2} G_{0}-G_{m+1} G_{1}\right)\right\}-h_{m}^{2} \\
= & (-1)^{n} a^{\tau(n)} b^{\xi(n+1)}\left\{\Delta_{m}-h_{m}(a b+2)\right\} G_{n} G_{n+m+1}+h_{m} a b\left(G_{m+1} G_{1}-G_{m+2} G_{0}\right)-h_{m}^{2} .
\end{aligned}
$$

If $n \in \mathbb{N}_{e}$, then there exists a positive integer $m_{0}$ such that, for $n \geq m_{0}, X_{1}<0$, and

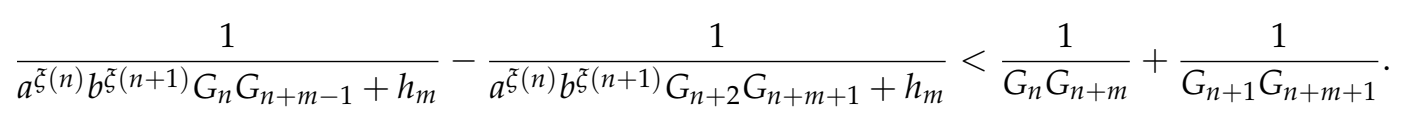

Repeatedly applying the above inequality, we have

$$
\frac{1}{a^{\xi(n)} b^{\tau(n+1)} G_{n} G_{n+m-1}+h_{m}}<\sum_{k=n}^{\infty} \frac{1}{G_{k} G_{k+m}} \text {, if } n \geq m_{0} \text { and } n \in \mathbb{N}_{e}
$$

Similarly, we obtain, for some positive integer $m_{1}$,

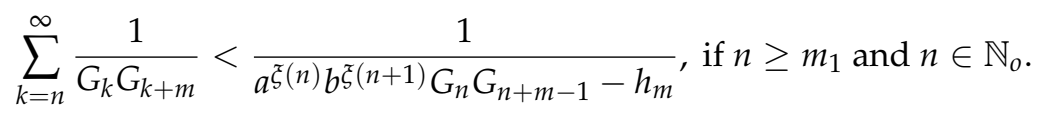

Next, consider

$$
\begin{aligned}
& X_{2}=\frac{1}{a^{\tau}(n) b^{\tau}(n+1) G_{n} G_{n+m-1}+(-1)^{n} h_{m}-1}-\frac{1}{a^{\tau(n+1)} b^{\tau}(n) G_{n+1} G_{n+m}+(-1)^{n+1} h_{m}-1}-\frac{1}{G_{n} G_{n+m}}
\end{aligned}
$$

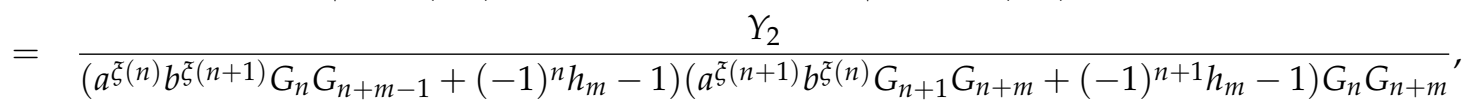

$$
\begin{aligned}
& Y_{2}=a^{\xi(n+1)} b^{\xi(n)} G_{n} G_{n+1} G_{n+m}^{2}-a b G_{n} G_{n+1} G_{n+m-1} G_{n+m}-a^{\xi(n)} b^{\tilde{\xi}(n+1)} G_{n}^{2} G_{n+m-1} G_{n+m+1} \\
& -(-1)^{n} h_{m}\left(2 G_{n} G_{n+m}+a^{\xi(n+1)} b^{\xi(n)} G_{n+1} G_{n+m}-a^{\xi(n)} b^{\xi(n+1)} G_{n} G_{n+m-1}\right) \\
& \left.+a^{\xi(n)} b^{\xi(n+1)} G_{n} G_{n+m-1}+a^{\xi(n+1)} b^{\xi(n)} G_{n+1} G_{n+m}\right)+h_{m}^{2}-1 \text {. }
\end{aligned}
$$

Using Lemma $2(\mathrm{~d}, \mathrm{e})$, we have

$$
\begin{aligned}
& a^{\xi(n+1)} b^{\xi(n)} G_{n} G_{n+1} G_{n+m}^{2}-a b G_{n} G_{n+1} G_{n+m-1} G_{n+m}-a^{\xi(n)} b^{\xi(n+1)} G_{n}^{2} G_{n+m-1} G_{n+m+1} \\
& =a^{\xi(n+1)} b^{\xi(n)} G_{n} G_{n+1} G_{n+m}\left(G_{n+m}-a^{\xi^{(n)}} b^{\tilde{\xi}(n+1)} G_{n+m-1}\right)-a^{\xi(n)} b^{\xi(n+1)} G_{n}^{2} G_{n+m-1} G_{n+m} \\
& =G_{n} G_{n+m}\left(a^{\xi(n+1)} b^{\xi(n)} G_{n+1} G_{n+m-2}-a^{\xi(n)} b^{\xi(n+1)} G_{n} G_{n+m-1}\right) \\
& =(-1)^{n}\left(a G_{m} G_{3}-b G_{m+1} G_{2}\right) G_{n} G_{n+m} \text {, }
\end{aligned}
$$

and

$$
\begin{aligned}
& \left.2 G_{n} G_{n+m}+a^{\xi(n+1)} b^{\xi(n)} G_{n+1} G_{n+m}-a^{\xi(n)} b^{\xi(n+1)} G_{n} G_{n+m-1}\right) \\
= & \left.2 G_{n} G_{n+m}+a^{\xi(n+1)} b^{\xi(n)}\left(a^{\xi(n)} b^{\xi(n+1)} G_{n}+G_{n-1}\right) G_{n+m}-a^{\xi(n)} b^{\xi(n+1)} G_{n} G_{n+m-1}\right) \\
= & \left.(a b+2) 2 G_{n} G_{n+m}+a^{\xi(n+1)} b^{\xi(n)} G_{n-1} G_{n+m}-a^{\xi(n)} b^{\xi(n+1)} G_{n} G_{n+m-1}\right) \\
= & (a b+2) 2 G_{n} G_{n+m}+(-1)^{n}\left(a G_{m} G_{1}-b G_{m+1} G_{0}\right) .
\end{aligned}
$$

Hence we obtain

$$
\begin{aligned}
Y_{2}= & \left.(-1)^{n}\left\{\Delta_{m}-h_{m}(a b+2)\right\} G_{n} G_{n+m}+a^{\xi(n)} b^{\xi(n+1)} G_{n} G_{n+m-1}+a^{\xi(n+1)} b^{\xi(n)} G_{n+1} G_{n+m}\right) \\
& -h_{m}\left(a G_{m} G_{1}-b G_{m+1} G_{0}\right)+h_{m}^{2}-1,
\end{aligned}
$$

and there exists a positive integer $m_{2}$ such that, for $n \geq m_{2}, X_{2}>0$, and 


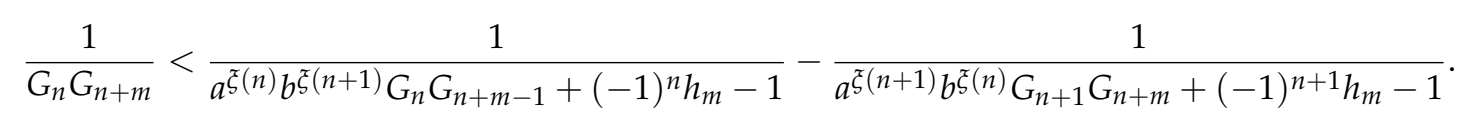

Repeatedly applying the above inequality, we have

$$
\sum_{k=n}^{\infty} \frac{1}{G_{k} G_{k+m}}<\frac{1}{a^{\tau(n)} b^{\tau(n+1)} G_{n} G_{n+m-1}+(-1)^{n} h_{m}-1} \text {, if } n \geq m_{2} .
$$

Finally, consider

$$
\begin{aligned}
& X_{3}=\frac{1}{a^{\tau}(n) b^{\tau}(n+1) G_{n} G_{n+m-1}+(-1)^{n} h_{m}+1}-\frac{1}{a^{\tau(n+1)} b^{\tau}(n) G_{n+1} G_{n+m}+(-1)^{n+1} h_{m}+1}-\frac{1}{G_{n} G_{n+m}} \\
& =\frac{Y_{3}}{\left(a^{\tau}(n) b \xi^{\tau}(n+1) G_{n} G_{n+m-1}+(-1)^{n} h_{m}+1\right)\left(a^{\tau}(n+1) b \xi^{\tau}(n) G_{n+1} G_{n+m}+(-1)^{n+1} h_{m}+1\right) G_{n} G_{n+m}}, \\
& \text { where } \\
& \begin{aligned}
Y_{3}= & Y_{2}-2\left(a^{\xi(n)} b^{\xi(n+1)} G_{n} G_{n+m-1}+a^{\xi(n+1)} b^{\xi(n)} G_{n+1} G_{n+m}\right) \\
= & \left.(-1)^{n}\left\{\Delta_{m}-h_{m}(a b+2)\right\} G_{n} G_{n+m}-a^{\xi(n)} b^{\xi(n+1)} G_{n} G_{n+m-1}-a^{\xi(n+1)} b^{\xi(n)} G_{n+1} G_{n+m}\right) \\
& -h_{m}\left(a G_{m} G_{1}-b G_{m+1} G_{0}\right)+h_{m}^{2}-1 .
\end{aligned}
\end{aligned}
$$

There exists a positive integer $m_{3}$ such that, for $n \geq m_{3}, X_{3}<0$, and

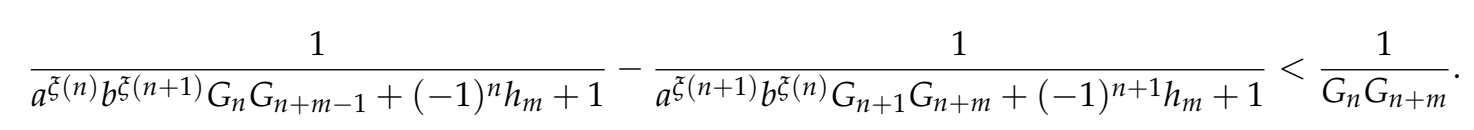

Repeatedly applying the above inequality, we have

$$
\frac{1}{a^{\tau(n)} b^{\tau(n+1)} G_{n} G_{n+m-1}+(-1)^{n} h_{m}+1}<\sum_{k=n}^{\infty} \frac{1}{G_{k} G_{k+m}}, \text { if } n \geq m_{2} .
$$

Then (19) follows from (23)-(26).

Now suppose that $\Delta_{m}<0$. In this case, we have

$$
\Delta_{m}-h_{m}(a b+2)>0,
$$

and (23)-(26) are respectively modified as

$$
\begin{aligned}
& \sum_{k=n}^{\infty} \frac{1}{G_{k} G_{k+m}}<\frac{1}{a^{\tau}(n) b^{\tau}(n+1) G_{n} G_{n+m-1}+h_{m}} \text {, if } n \geq m_{4} \text { and } n \in \mathbb{N}_{e}, \\
& \frac{1}{a^{\tau}(n) b^{\tau}(n+1) G_{n} G_{n+m-1}-h_{m}}<\sum_{k=n}^{\infty} \frac{1}{G_{k} G_{k+m}} \text {, if } n \geq m_{5} \text { and } n \in \mathbb{N}_{o} \text {, } \\
& \sum_{k=n}^{\infty} \frac{1}{G_{k} G_{k+m}}<\frac{1}{a^{\tau(n)} b^{\tau(n+1)} G_{n} G_{n+m-1}+(-1)^{n} h_{m}-1}, \text { if } n \geq m_{6},
\end{aligned}
$$

and

$$
\frac{1}{a^{\tau(n)} b^{\tau(n+1)} G_{n} G_{n+m-1}+(-1)^{n} h_{m}+1}<\sum_{k=n}^{\infty} \frac{1}{G_{k} G_{k+m}}, \text { if } n \geq m_{7} .
$$

Then, (20) easily follows and the proof of (a) is completed.

(b) (21) and (22) can be proved as in Theorem 1, and details are omitted.

If $a=b$, then Theorem 2 reduces ([7], (Theorem 2.1) with $m \in \mathbb{N}_{o}$ and $\left\{G_{n}\right\}=\left\{H_{n}\right\}=$ $S\left(G_{0}, G_{1}, a, a\right)$. 


\section{Discussion}

This paper concerned the properties of the generalized bi-periodic Fibonacci numbers $\left\{G_{n}\right\}$ generated from the recurrence relation: $G_{n}=a G_{n-1}+G_{n-2}$ ( $n$ is even) or $G_{n}=b G_{n-1}+G_{n-2}$ ( $n$ is odd). We derived quite a general identities related to reciprocal sums of products of two generalized bi-periodic Fibonacci numbers. More precisely, we obtained formulas for the integer parts of the numbers

$$
\left(\sum_{k=n}^{\infty} \frac{\left(\frac{a}{b}\right)^{\xi(k+1)}}{G_{k} G_{k+m}}\right)^{-1}, m=0,2,4, \cdots,
$$

and

$$
\left(\sum_{k=n}^{\infty} \frac{1}{G_{k} G_{k+m}}\right)^{-1}, m=1,3,5, \cdots .
$$

The identities obtained in this paper include many existing results as special cases. As already noted in [16], an open problem is whether we can obtain similar results for the same numbers of higher order. It seems that we can also derive similar identities for the numbers of the form

$$
\left(\sum_{k=n}^{\infty} \frac{1}{G_{k} H_{k+m}}\right)^{-1}, m=0,1,2,3, \cdots,
$$

where $\left\{G_{n}\right\}=S\left(G_{0}, G_{1}, a, b\right)$ and $\left\{H_{n}\right\}=S\left(H_{0}, H_{1}, a, b\right)$, which is left as another open problem.

Funding: This research received no external funding.

Institutional Review Board Statement: Not applicable.

Informed Consent Statement: Not applicable.

Data Availability Statement: Not applicable.

Conflicts of Interest: The author declares no conflict of interest.

\section{References}

1. Koshy, T. Fibonacci and Lucas Numbers with Applications; Wiley: New York, NY, USA, 2001.

2. Falcon, S.; Plaza, A. On the Fibonacci $k$-numbers. Chaos Solitons Fractals 2007, 32, 1615-1624. [CrossRef]

3. Edson, M.; Yayenie, O. A new generalization of Fibonacci sequence and extended Binet's formula. Integers 2009, 9, 639-654. [CrossRef]

4. Filipponi, P. Incomplete Fibonacci and Lucas numbers. Rend. Circ. Mat. Palermo 1996, 45, 37-56. [CrossRef]

5. Ramírez, J.L. Bi-periodic incomplete Fibonacci sequences. Ann. Math. Inform. 2013, 42, 83-92.

6. Ohtsuka, H.; Nakamura, S. On the sum of reciprocal Fibonacci numbers. Fibonacci Quart. 2008, 46, 153-159.

7. Choo, Y. On the reciprocal sums of products of two generalized Fibonacci numbers. Int. J. Math. Anal. 2019 13, 539-549. [CrossRef]

8. Choi, G.; Choo, Y. On the reciprocal sums of products of Fibonacci and Lucas numbers. Filomat 2018, 32, 2911-2920. [CrossRef]

9. Holliday, S.; Komatsu, T. On the sum of reciprocal generalized Fibonacci numbers. Integers 2011, 2011, 11. [CrossRef]

10. Kuhapatanakul, K. On the sums of reciprocal generalized Fibonacci numbers. J. Integer Seq. 2013, 16, 3.

11. Liu, R.; Wang, A. Sums of products of two reciprocal Fibonacci numbers. Adv. Differ. Equ. 2016, 2016, 1136. [CrossRef]

12. Wang, A.; Wen, P. On the partial finite sums of the reciprocals of the Fibonacci numbers. J. Inequal. Appl. 2015, 15, 73. [CrossRef]

13. Wang, A.; Yuan, T. Alternating sums of the reciprocal Fibonacci numbers. J. Integer Seq. 2017, 20, 17.1.4.

14. Wang, A.; Zhang, F. The reciprocal sums of even and odd terms in the Fibonacci sequence. J. Inequal. Appl. 2015, 2015, 376. [CrossRef]

15. Zhang, W.; Wang, T. The infinite sum of reciprocal Pell numbers. Appl. Math. Comput. 2012, $218,6164-6167$.

16. Basbuk, M.; Yazlik, Y. On the sum of reciprocal of generalized bi-periodic Fibonacci numbers. Miskolc Math. Notes 2016, $17,35-41$. [CrossRef]

17. Choi, G.; Choo, Y. On the reciprocal sums of square of generalized bi-periodic Fibonacci numbers. Miskolc Math. Notes 2018, 19, 201-209. [CrossRef]

18. Choo, Y. On the generalizations of Fibonacci identities. Results Math. 2017, 71, 347-356. [CrossRef] 